\title{
FRAGILIZAÇÃO POR HIDROGÊNIO EM BARRAS DE AÇO DE ALTA RESISTÊNCIA: UM ESTUDO DE CASO*
}

\author{
Alex Maia do Nascimento ${ }^{1}$ \\ Rogerio Ermelindo de Souza ${ }^{2}$ \\ Sandro Gonçalves Silva ${ }^{3}$
}

\section{Resumo}

Durante a produção de aços em fornos elétricos a arco (FEA), o hidrogênio pode ser incorporado ao banho líquido através de diversas formas: umidade das matériasprimas, umidade do ar (no momento de abertura do arco elétrico), umidade dos refratários e etc. A fragilização por hidrogênio $(\mathrm{FH})$ é um fenômeno decorrente da incorporação e retenção de hidrogênio, acima de certo nível tolerável para cada aço em particular, que gera altíssimas tensões internas e diminui, catastroficamente, a capacidade de deformação do material. O objetivo do presente trabalho foi encontrar a quantidade de hidrogênio (ppm) aceitável no aço inicialmente desenvolvido para fabricação de barras CA-50 (6,3 / 8,0 / 10,0 e 12,5 mm), obtidas em rolos por laminação a quente após instalação de uma nova máquina de lingotamento contínuo de tarugos. Para realização do trabalho foram realizados ensaios de dobramento a $180^{\circ}$ conforme a norma NBR 7480/2007 e medições dos teores de Hidrogênio no distribuidor da Aciaria através do equipamento Hydris.

Palavras-chave: Fragilização por hidrogênio; Aços de alta resistência; Lingotamento contínuo.

\section{HYDROGEN EMBRITTLEMENT IN HIGH STRENGTH STEEL REBARS - A CASE STUDY}

\section{Abstract}

During the steel production in electric arc furnaces (EAF), the hydrogen can be incorporated into the liquid bath through: humidity from raw materials, air humidity (electric arc opening), humidity from refractories and etc. The hydrogen embrittlement $(\mathrm{HE})$ is a phenomenon due to the incorporation and retention of hydrogen above a certain tolerable level for each particular steel, which generates extremely high internal stress and catastrophically decreases the ductility of the material. This study aims to find the amount of hydrogen (measured in ppm) acceptable in the original steel developed for the production of CA-50 bars (6.3/8.0 / 10.0 and $12.5 \mathrm{~mm})$, obtained in hot rolling after installation of a new machine of billet continuous casting. Bending tests were performed according to NBR 7480/2007 standard and hydrogen content measurements were taken from the tundish using Hydris equipment.

Keywords: Hydrogen embrittlement; High strength steels; Continuous casting. 


\section{INTRODUÇÃO}

$\mathrm{Na}$ indústria siderúrgica, o assunto de fratura retardada em aços de alta resistência torna-se um assunto importante porque geralmente a susceptibilidade à fragilização por hidrogênio $(\mathrm{FH})$ aumenta com o aumento da resistência dos aços [1]. A Tabela 1 mostra a susceptibilidade à $\mathrm{FH}$ em relação a diversas classes de materiais [2], onde se observa que aços com alta resistência e alto limite de escoamento possuem alta susceptibilidade à $\mathrm{FH}$.

Tabela 1. Ranking das classes de materiais mais susceptíveis à $\mathrm{FH}$ [adaptado de 2].

\begin{tabular}{|l|l|}
\hline \multicolumn{1}{|c|}{$\begin{array}{c}\text { Susceptibilidade à FH } \\
\text { (Ranking em ordem } \\
\text { decrescente) }\end{array}$} & \multicolumn{1}{|c|}{ Material } \\
\hline Alta & \begin{tabular}{l} 
Aços de alta resistência e alto limite de \\
escoamento \\
\hline Ligas de Níquel de alta resistência \\
Aços de média e de baixa resistência \\
Monocristais de Ferro-Silício \\
Ligas de Cobalto
\end{tabular} \\
\hline Baixa & Aços inoxidáveis austeníticos \\
\hline Pequena ou nula & $\begin{array}{l}\text { Aços inoxidáveis estabilizados } \\
\text { Ligas de Alumínio } \\
\text { Ligas de Molibdênio }\end{array}$ \\
\hline
\end{tabular}

O hidrogênio induz efeitos intrinsicamente fragilizantes [3]. A presença de hidrogênio em uma variedade de aços resulta na degradação das suas propriedades mecânicas e a fragilização é manifestada pela diminuição da ductilidade e da tensão verdadeira de fratura [3-5].

O hidrogênio após ser incorporado durante fabricação do aço (via umidade das matérias-primas, refratários, durante abertura do arco, etc) poderá, após etapa de solidificação, ser difundido para fora do produto. A cinética de difusão dependerá da taxa de resfriamento, quanto menor a taxa de resfriamento, maior será a difusividade, devido ao fato que o hidrogênio possui alta mobilidade e esta cresce exponencialmente com a temperatura [6].

A Figura 1 ilustra a difusividade do hidrogênio versus temperatura para materiais austeníticos (CFC) e ferríticos (CCC). Observa-se que a difusividade do hidrogênio na ferrita é superior a difusividade na austenita, além do que abaixo de $200^{\circ} \mathrm{C}$ a difusão cai drasticamente para a ferrita e é praticamente desprezível para materiais austeníticos [8].

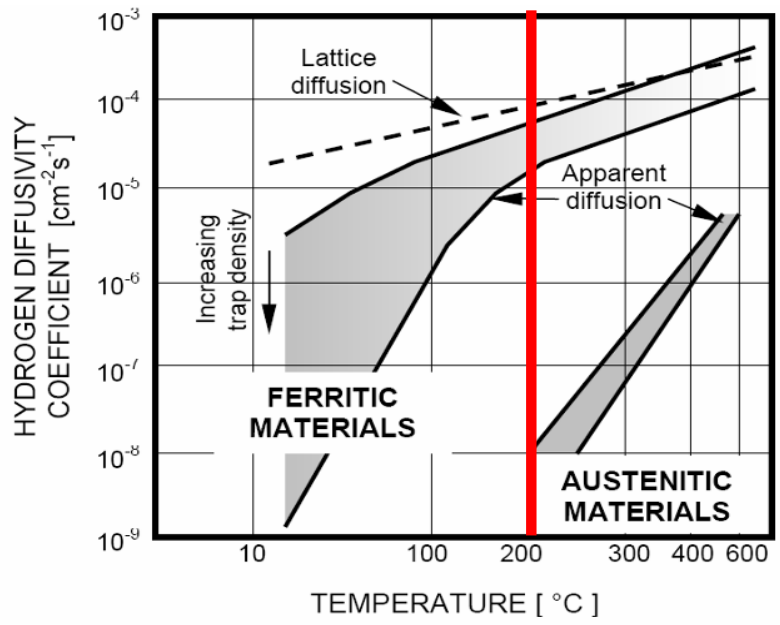

Figura 1. Difusão do hidrogênio em relação à temperatura e estrutura cristalina [8]. 
Em função da teoria abordada anteriormente, a manutenção das temperaturas dos tarugos de aços na faixa de $200-700{ }^{\circ} \mathrm{C}$ por maior tempo facilita a dessorção do hidrogênio, evitando assim a sua segregação para o centro do tarugo. Uma forma de promover a dessorção de hidrogênio utilizada por muitas empresas é realizar o empilhamento dos tarugos em temperaturas em torno de $700^{\circ}-900^{\circ} \mathrm{C}$, logo após o lingotamento. Essa técnica é considerada bastante eficiente. A Figura 2 ilustra tarugos empilhados em locais fechados proporcionando baixas taxas de resfriamento, consequentemente altas taxas de dessorção de hidrogênio.

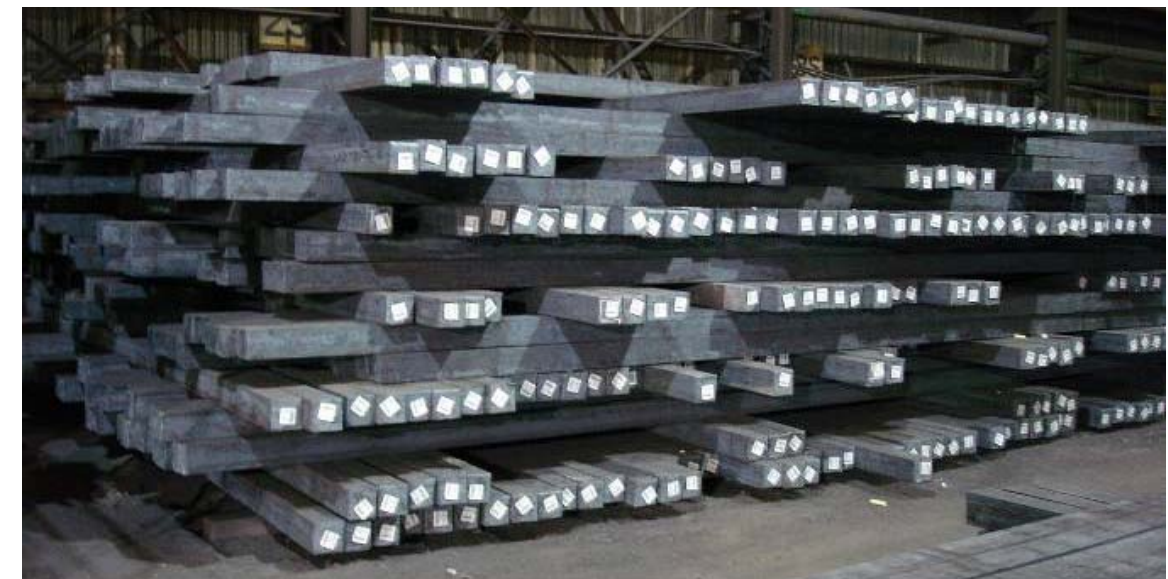

Figura 2. Empilhamento de tarugos visando remoção de Hidrogênio em local fechado.

Os aços utilizados para produção de vergalhão CA-50 em rolo, devem apresentar valores elevados de limite de escoamento (LE) e de resistência (LR), além de apresentar uma boa ductilidade, característica essa que pode ser comprometida devido ao teor de hidrogênio presente no aço.

A norma NBR 7480/2008 especifica as propriedades mecânicas à tração e dobramento (ductilidade na fibra externa sem aparecimento de fissuras) de acordo com a bitola do vergalhão CA-50.

O objetivo do presente trabalho foi encontrar a teor de hidrogênio aceitável no aço utilizado para produção de barras de aço CA 50 (6,3/8,0/10,0 e 12,5mm), obtidas em rolos por laminação a quente, em função das taxas de resfriamento dos tarugos.

\section{MATERIAIS E MÉTODOS}

O \%C do aço utilizado na realização do presente trabalho está ilustrada na Tabela 2, bem como o valor de carbono equivalente que consiste em um número que expressa de forma simples e objetiva a susceptibilidade à $\mathrm{FH}$. A Tabela 3 sumariza a susceptibilidade à $\mathrm{FH}$ em relação ao carbono equivalente [7]. Com base nas Tabelas apresentadas anteriormente o aço desenvolvido para eventual produção das barras de aço CA-50 possui uma alta susceptibilidade à $\mathrm{FH}$.

Tabela 2. \%C do aço (\% em peso) e o valor de carbono equivalente.

$$
\begin{array}{|c|c|}
\hline \text { \%C } & \mathbf{C}_{\text {eq }}{ }^{*} \\
\hline \mathbf{0 , 4 8} & \mathbf{0 , 6 7} \\
\hline
\end{array}
$$


Tabela 3. Susceptibilidade à FH versus Carbono Equivalente [7].

\begin{tabular}{|c|c|}
\hline $\begin{array}{c}\text { Susceptibilidade à } \\
\text { FH }\end{array}$ & $\begin{array}{c}\text { Carbono } \\
\text { Equivalente }\end{array}$ \\
\hline Baixa & $\mathrm{C}_{\mathrm{eq}}<0,40$ \\
\hline Média & $0,40 \leq \mathrm{C}_{\mathrm{eq}}<0,60$ \\
\hline Alta & $\mathrm{C}_{\mathrm{eq}}>0,60$ \\
\hline
\end{tabular}

Os ensaios de dobramento foram realizados conforme ilustra a Figura 3. A norma NBR 7480:2007 exige para barras de aço de bitolas inferiores a $20 \mathrm{~mm}$ que o dobramento seja realizado em pinos com diâmetro igual a 4x o diâmetro da bitola do vergalhão.

Visando tornar o dobramento mais severo, realizou-se no presente trabalho ensaios de dobramento com diâmetro do pino igual a $2 x$ o diâmetro do vergalhão. Após o dobramento foi observado a presença ou ausência de fissuras/quebras e foi quantificado o índice de desclassificação (ID). O ID consistiu da relação entre o número de amostras que apresentavam fissuras/quebras em relação aos 50 ensaios realizados on-line para cada bitola (logo em seguida à produção).
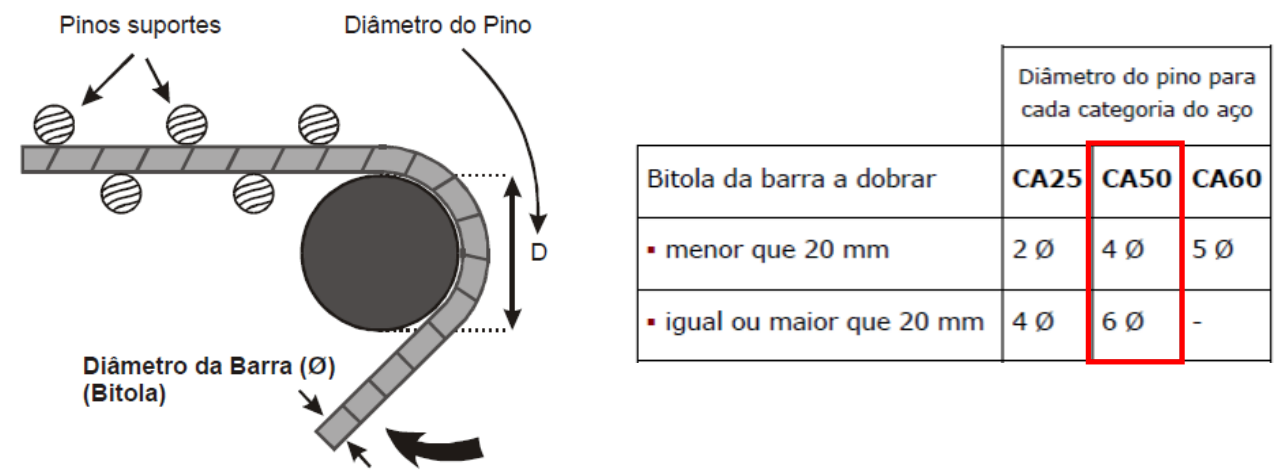

Figura 3. Diagrama esquemático do ensaio de dobramento realizado nos vergalhões.

O teor de hidrogênio foi medido pelo Sistema Hydris no distribuidor. O método consiste em medir a pressão gasosa de $\mathrm{H}_{2}$ existente no aço líquido. Baseado na constante de equilíbrio entre a fase gasosa e o aço líquido e a lei de Sievert, é possível correlacionar o teor de gás dissolvido para a composição de gás conhecida. A medição gira em torno de 1 minuto e possui uma precisão excelente. O sistema Hydris atualmente é um padrão mundial para análises e certificação de hidrogênio. $A$ medição foi realizada em diversas corridas do aço em questão, e dentro de cada corrida foram destinados tarugos para as diferentes bitolas de barras de modo a se verificar a aprovação ou não no teste de dobramento para o respectivo teor de hidrogênio medido.

\section{RESULTADOS E DISCUSSÃO}

As medições dos teores de hidrogênio foram realizadas em distribuidores de dois diferentes lingotamentos contínuos (LC). Isso possibilitou a verificação do efeito da taxa de resfriamento na dessorção do hidrogênio, após a solidificação. A Figura 4 ilustra os dois leitos de resfriamento. Observa-se que no LC_A os tarugos depois de solidificados trocam calor por condução o que minimiza a taxa de resfriamento, além do que o resfriamento ocorre em local fechado isento da ação de intempéries (chuvas e ventos fortes). Já no LC_B não há troca de calor entre os tarugos, e o leito 
de resfriamento é descoberto, o que ocasiona altas taxas de resfriamento em comparação ao LC_A. Como abordado anteriormente, altas taxas de resfriamento reduzem a dessorção de hidrogênio, resultando em uma migração de hidrogênio para o centro do tarugo.

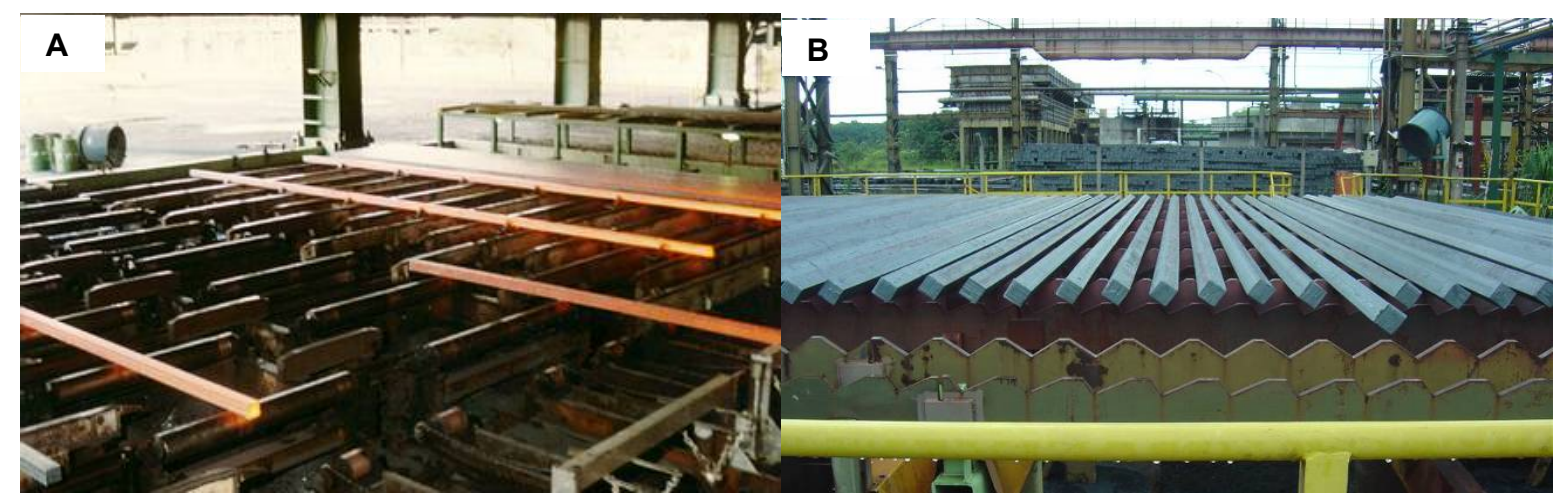

Figura 4. Leitos de resfriamento dos lingotamentos contínuos: A) taxa de resfriamento baixa e B) taxa de resfriamento alta.

As curvas de resfriamento de ambos LCs foram levantadas conforme apresentado na Figura 5a. Com o levantamento realizado pode-se quantificar as taxas de resfriamento para cada LC. Nota-se que em ambos os LCs a temperatura inicial medida no leito de resfriamento foi de aproximadamente $800^{\circ} \mathrm{C}$. As medições foram realizadas até os tarugos apresentarem temperatura superficial de $200^{\circ} \mathrm{C}$, devido a faixa de maior difusividade do hidrogênio ser entre $200^{\circ}-700^{\circ} \mathrm{C}$. Com os dados levantados realizou-se uma interpolação quadrática como exposto nas Figuras $5 \mathrm{~b}$ e 5c para ambos LCs, o que permitiu a obtenção da taxa de resfriamento em relação ao tempo.

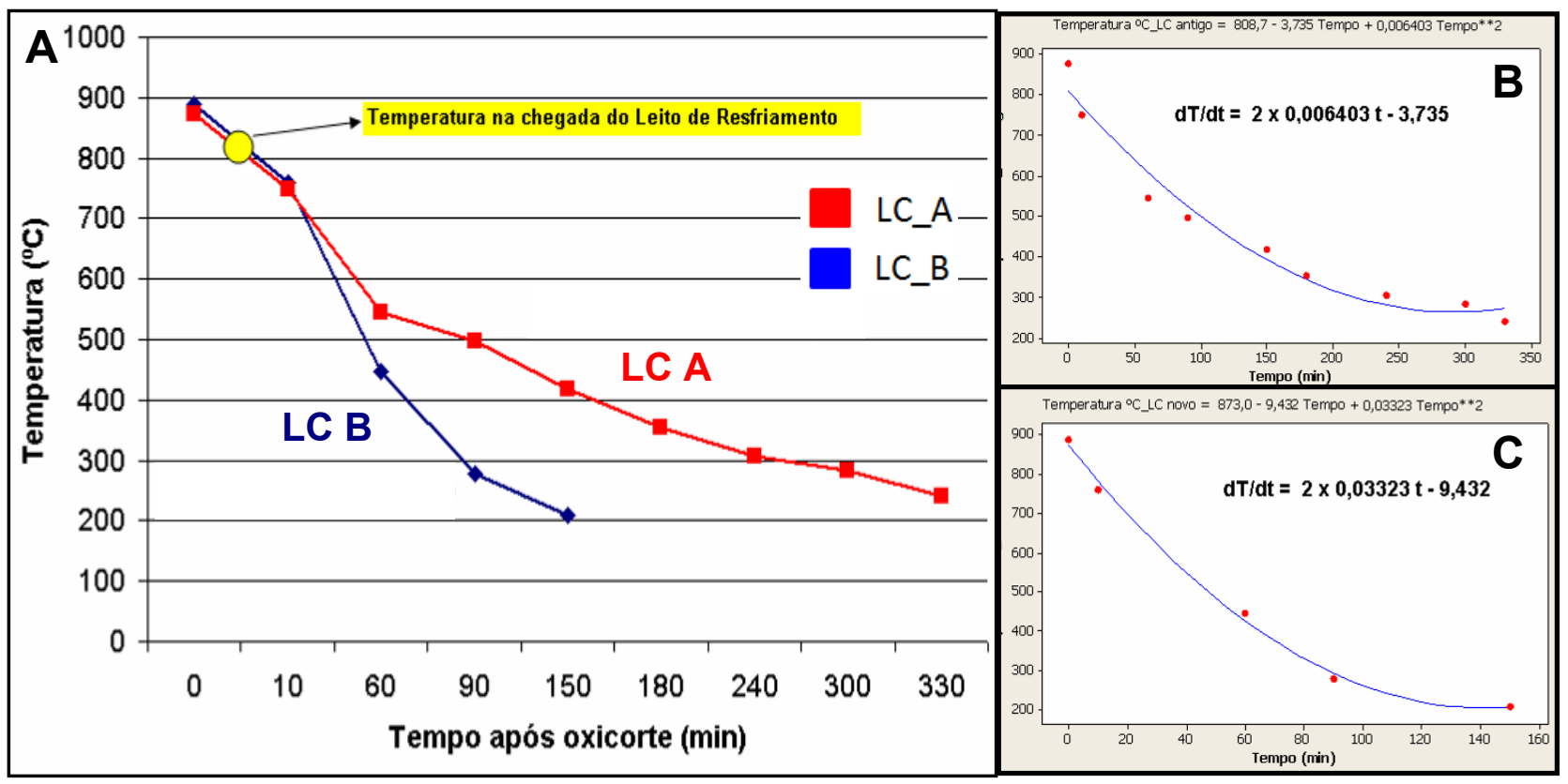

Figura 5. A) Curvas de resfriamento levantadas por pirômetro dos lingotamentos contínuos $A$ e $B$; B) Interpolação quadrática dos pontos levantados no LC_A - taxa de resfriamento baixa e C) Interpolação quadrática dos pontos levantados no LC_B - taxa de resfriamento alta.

A Figura 6 apresenta as taxas de Resfriamento dos LCs com o passar do tempo, observa-se que o LC_A apresenta praticamente uma taxa de resfriamento constante 
de $3{ }^{\circ} \mathrm{C} / \mathrm{min}$ durante todo o resfriamento. Já o LC B possui inicialmente taxas de resfriamento bem superiores, chegando a apresentar taxas próximas de $9{ }^{\circ} \mathrm{C} / \mathrm{min}$ no início de resfriamento. Observa-se também que durante os 90 minutos iniciais o LC B sempre apresentou taxas de resfriamento superiores, o que acarreta numa menor difusividade do hidrogênio. Observa-se uma grande variação da taxa de resfriamento no LC_B, enquanto o LC_A apresenta uma pequena taxa de resfriamento além de ser praticamente constante.

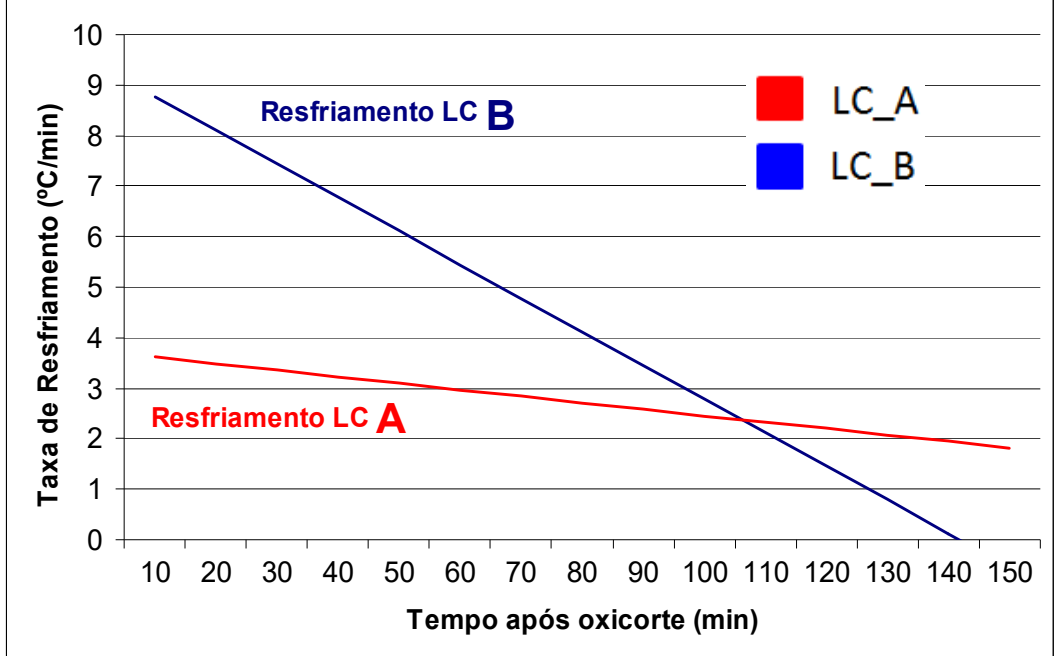

Figura 6. Taxas de resfriamento dos LCs A e B.

Utilizando o Equipamento Hydris foram medidos os teores de hidrogênio presentes em 50 diferentes corridas. As medições foram realizadas nos distribuidores dos lingotamentos contínuos. O aço solidificado no LC_A não apresentou problema de $\mathrm{FH}$, considerando como fator responsável a baixa taxa de resfriamento. Já o aço solidificado no LC_B apresentou a existência do fenômeno de FH.

A Figura 7 apresenta o valor de Hidrogênio utilizando o equipamento Hydris. O valor médio foi de 6 ppm nas medições realizadas no distribuidor da rota LC_B. O valor mínimo e máximo medido foi de 5,6 - 8,8 ppm, respectivamente.

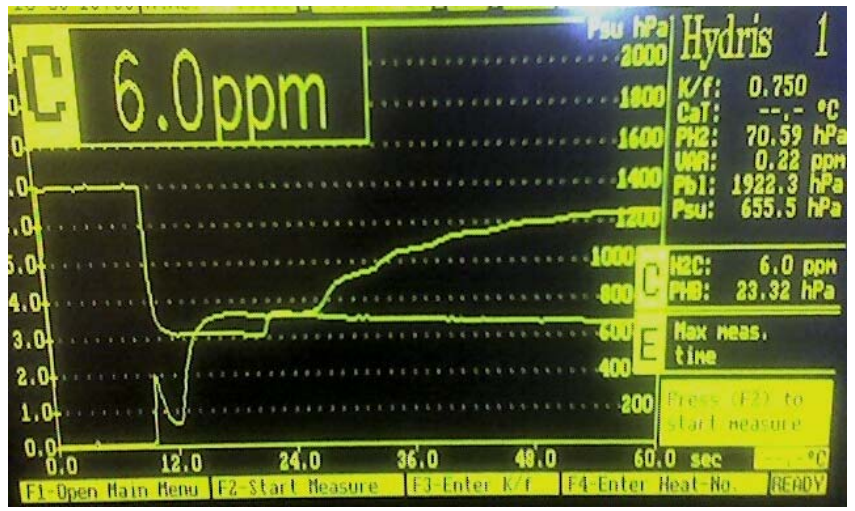

Figura 7. Medição do teor de hidrogênio presente no aço líquido utilizando Equipamento Hydris.

Cada corrida produziu 100 tarugos que foram divididos para serem utilizados nas 4 bitolas $(6,30$ / 8,00 / 10,0 e 12,5 mm). Após a laminação à quente, os ensaios de tração e dobramento foram realizados nas diferentes bitolas. O ensaio de dobramento demonstrou ser uma boa ferramenta para detecção do fenômeno de FH. Pelos ensaios de tração não foi possível verificar alterações nas propriedades 
mecânicas de limite de escoamento e resistência, mas observou-se uma diminuição no alongamento e na redução de área.

Após laminação foram realizados 100 ensaios de dobramento para cada bitola, com o objetivo de encontrar o ID (índice de desclassificação) devido ao aparecimento de fissura/quebra. O ID é uma ferramenta usada nas empresas, onde se busca visualizar o não atendimento a uma especificação através da quantificação percentual. A Figura 8a ilustra o sumário do ID para as diferentes bitolas, enquanto o teor de hidrogênio aceitável para cada bitola é apresentado na Figura 8b. O CA-50 de bitola $12,5 \mathrm{~mm}$ apresentou ID $=100 \%$, o que torna a produção crítica dessa bitola usando o processo do LC_B com o aço em questão. Utilizando a $2^{\mathrm{a}}$ lei de Fick e, considerando o coeficiente de difusão do hidrogênio de $2,5 \times 10^{-11} \mathrm{~m}^{2} / \mathrm{s}$ [9], pôde-se calcular o tempo necessário para dessorção do Hidrogênio existente. Foi considerado o teor de Hidrogênio não prejudicial na superfície da barra de 2 ppm e o valor no centro igual a 8 ppm (condição crítica). Após os cálculos para a bitola de $12,5 \mathrm{~mm}$ encontrou-se um valor em torno de 7 dias para realização de retestes. Após esse período, os retestes foram realizados e constatou-se a aprovação $100 \%$ das barras de aço ao ensaio de dobramento. Para evitar custos com material parado no estoque e eventuais atrasos em entregas foi desenvolvido um aço com $0,30 \% \mathrm{C} \mathrm{e}$ Ceq<0,55 para a Máquina de LC_B de forma a garantir a liberação $100 \%$ on-line de todas as bitolas. Maiores detalhes do presente desenvolvimento do aço para o LC_B serão apresentados posteriormente em outro congresso.
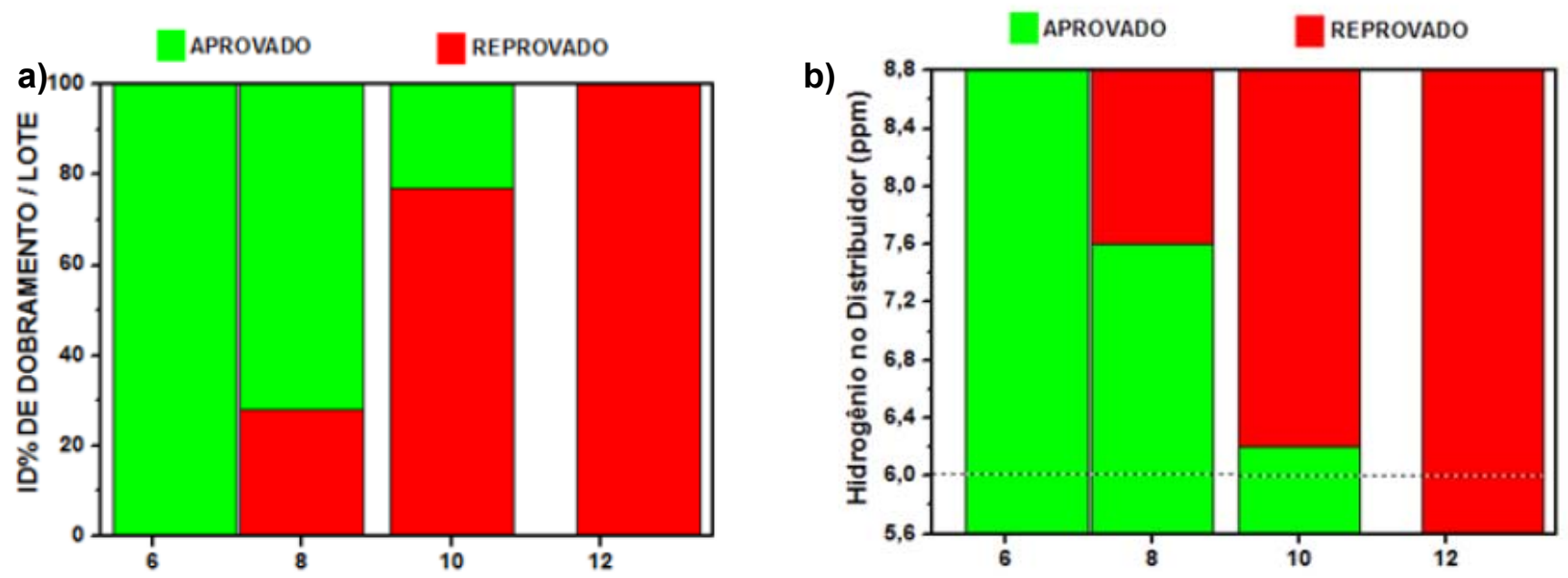

Figura 8. a) Índice de Desclassificação no Ensaio de Dobramento para as diferentes bitolas e b) Teor de Hidrogênio aceitável (crítico) para produção do CA-50 para cada bitola via processo LC B.

\section{CONCLUSÃO}

O LC_A apresentou $100 \%$ de aprovação no ensaio de dobramento com o aço de $0,48 \% \mathrm{C}$ e Ceq $>0,60$, devido a maior dessorção do hidrogênio oriundo da menor taxa de resfriamento propiciada.

O LC_B que propicia altas taxas de resfriamento apresentou a existência do fenômeno de $\mathrm{FH}$ quando utilizando aço com $0,48 \% \mathrm{C}$ e Ceq $>0,60$. Foi necessário o desenvolvimento de um aço com $0,30 \% \mathrm{C}$ e Ceq<0,55 (maior \% de ferrita próeutetóide) para evitar a $\mathrm{FH}$ via rota $\mathrm{LC}_{-} \mathrm{B}$, o que acarretou na liberação on-line de todos produtos/bitolas.

A influência da energia de deformação da laminação no processo de dessorção de hidrogênio é significativa, pois foi perceptível que o índice de desclassificação ID (\%) 
aumenta para as bitolas mais grossas. Quanto maior a bitola da barra de aço mais susceptível ao fenômeno $\mathrm{FH}$ e mais dias são necessários para dessorção de hidrogênio.

Para as barras CA-50 $6,3 \mathrm{~mm}, 8 \mathrm{~mm}, 10 \mathrm{~mm}$, os valores aceitáveis (máximo permitido) de Hidrogênio no distribuidor do LC_B são 8,8ppm, 7,6 ppm e 6,2ppm, respectivamente. A solidificação via rota $L C \_\bar{B}$ não propicia teores de Hidrogênio aceitáveis para aprovação da barra CA-50 12,5 mm no teste de dobramento on-line com a composição do aço \%0,48C e Ceq>0,67.

\section{REFERÊNCIAS}

1 Prosanto, M. E., Brizuela, G., Juan, A., "The Electronic Structure and Location of H Pairs in bcc Fe Edge Dislocation”, Applied Surface Science, v. 173, pp. 378 - 379, 2001.

2 Smithell Metals Reference Book, Butterworths \& Co. Publishing, $6^{\text {th }}$ edition, 1983.

3 Eliaz et al., Characteristics of Hydrogen Embrittlement, Stress Corrosion Cracking and Tempered Martensite Embrittlement in High-Strenght Steels, Engineering Failure Analyses, Vol.9, edição 2, p 167-184, 2002.

4 Tiwari, G. P. et al., "A Study of Internal Hydrogen Embrittlement of Steels", Materials Science and Engineering, v. A286, pp. 269-281, 2000.

5 Zielinski, A., "Effect of Hydrogen on Internal Friction of Some F.C.C. Metals", Acta Metallurgical Materials, v. 38, n. 12, pp. 2573-2582, 1990.

6 Konkol, P.J. 2003, Effects of Long-Time Postweld Heat Treatment on the Properties of Constructional-Steel Weldments" Welding Research Council Bulletin 302, February 1988

7 Lancaster, J.F., "Metallurgy of Welding" Sixth Ed., 256-268, Woodhead Publishing, Abington Cambridge, England (1999).

8 Pitrun, M., "The Effect of Welding Parameters on Levels of Diffusible Hydrogen in Weld Metal Deposited Using Gas Shielding Rutile Flux Cored Wires", doctorate thesis, The University of Wollongong, Dpt. of Materials Engineering (2004).

$9 \mathrm{Kim}, \mathrm{J}$. S. et al., Effect Intergranular Ferrite on Hydrogen Delayed Fracture Resistance of Ultrahigh Strength Boron-added Steel, ISIJ International, v. 47, n. 6, pp. 913-919, 2007. 